\title{
Co-creation of an ICT-supported cancer rehabilitation program for lung cancer survivors
}

\author{
J.G. Timmerman ${ }^{1,2}$, T.M. Tönis ${ }^{1,2}$, M.M. Stuiver ${ }^{3}$, M.G.H. Dekker-van Weering ${ }^{1}$, M.W.J.M. Wouters ${ }^{3}$, H. \\ J. Hermens ${ }^{1,2}$, M.M.R. Vollenbroek-Hutten ${ }^{1,2}$ \\ ${ }^{1}$ Roessingh Research and Development, Telemedicine Research Group, Enschede, The Netherlands \\ ${ }^{2}$ University Twente, Faculty of Electrical Engineering, Biomedical Signals and Systems, Telemedicine \\ group, Enschede, The Netherlands \\ ${ }^{3}$ Netherlands Cancer Institute, Amsterdam, The Netherlands
}

Background: Lung cancer (LC) patients experience high symptom burden and significant decline of physical fitness and Quality of Life following lung resection. Good quality of survivorship care postsurgery is essential to optimize recovery and prevent unscheduled healthcare use. The use of Information and Communication Technology (ICT) can improve post-surgery care, as it enables frequent monitoring of health status in daily life, provides timely and personalized feedback to patients and professionals, and improves accessibility to rehabilitation programs. Despite its promises, implementation of ICT applications is challenging, often hampered by non-acceptance of the developed service by its end-users. A promising approach is to involve the end-users early and continuously during the developmental process through a so-called user-centred design approach. The aim of this abstract is to report on this process of co-creation of an ICT-supported cancer rehabilitation program with and for lung cancer patients and their healthcare professionals (HCP's).

Material and Methods: Requirements were established during semi-structured interviews and focus groups with LC patients $(n=10)$ and HCP's $(n=11)$ and validated by means of written scenarios describing the integration of the future program in existing healthcare processes. Requirement specification was succeeded by the development of a first prototype. Usability of the prototype was then evaluated with healthy subjects $(n=13), L C$ patients $(n=7)$ and HCP's $(n=9)$ by means of semistructured interviews and the System Usability Scale (SUS).

Results: The developed rehabilitation program contains the following modules: 1) symptom selfmonitoring of pain, dyspnoea, fatigue, and physical condition using on-body sensors ( $\mathrm{HR}, \mathrm{O}_{2}$ saturation, and accelerometer) in combination with a smartphone, and 2) an online physical exercise program. The results of the symptom monitoring module are visualised on a web portal integrating monitoring data with electronic patient records. Usability of the program was scored acceptable by $84 \%$ of the users (SUS median score $=78$ ). Overall, LC patients and HCP's found the system simple and effective for daily symptom monitoring and improvement of physical fitness with a home-based exercise program, and $73 \%$ would be willing to use the system when offered to them as part of lung cancer treatment.

Conclusion: With a user-centred design approach we were able to assess and evaluate the requirements of LC patients and HCP's for the ICT-supported rehabilitation program repeatedly during the entire developmental process, resulting in a final version of the program that matches the end users' requirements. The next step is to evaluate feasibility and clinical effect of the program as part of regular care following lung cancer surgery. 